Please do not remove this page

RMIT

UNIVERSITY

\title{
Millimetre-wave InP antenna for monolithic integration
}

Rowe, Wayne; Ghorbani, Kamran

https://researchrepository.rmit.edu.au/esploro/outputs/9921862849401341/filesAndLinks?institution=61RMIT_INST\&index=null

Rowe, W., \& Ghorbani, K. (2006). Millimetre-wave InP antenna for monolithic integration. Electronics Letters, 42(13), 733-734. https://doi.org/10.1049/el:20061253

Published Version: https://doi.org/10.1049/el:20061253

Repository homepage: https://researchrepository.rmit.edu.au

(c) IEE 2006. Personal use of this material is permitted. However, permission to reprint/republish this material for advertising or promotional purposes or for creating new collective works for resale or redistribution to servers or lists, or to reuse any copyrighted component of this work in other works must be obtained from the IEEE.

Downloaded On 2023/04/26 19:54:22 +1000 


\section{Millimetre-wave InP antenna for monolithic integration}

\section{W.S.T. Rowe and K. Ghorbani}

\begin{abstract}
A printed antenna realised on an indium phosphide wafer is presented. The antenna provides an efficient, broadband antenna solution for MMIC/OEIC systems and subsystems. The antenna is based on an aperture coupled patch architecture with the substrates layers in a hi-lo configuration. Measured results exhibit an impedance bandwidth of around $12 \%$ for $\mathrm{VSWR}<2$, and a gain of $8 \mathrm{dBi}$.
\end{abstract}

Introduction: Millimetre-wave fibre wireless applications [1] could profit from a small antenna that can be fully integrated with the microwave and optical components of the system. This can lead to smaller and more cost-effective fibre-fed antenna units. Active integrated antennas [2] are another application that could benefit from a directly integrated antenna.

Printed antennas are the obvious choice for monolithic integration, owing to their low profile, planar characteristics. However, the materials used to fabricate the microwave/optical devices (e.g. gallium arsenide or indium phosphide $(\operatorname{InP}))$ are typically of high permittivity $\left(\varepsilon_{r}>10\right)$. The design of conventional printed antenna elements on such substrates is generally avoided, as they suffer from a narrow bandwidth $(<6 \%)$ and excessive loss due to surface-wave excitation. To ensure that the antenna is not a limiting factor in the system bandwidth, wideband antenna elements are desired. Most of the MMIC/OEIC integratable antennas presented in the literature (e.g. [3]) exhibit a narrow bandwidth and/or indicators of poor efficiency. A coplanar waveguide (CPW)-fed aperture stacked patch (ASP) [4] has exhibited excellent performance on high dielectric constant feed materials by using a combination of high and low permittivity layers, similar to that described in [5].

Material systems based on InP have the potential to realise the active and passive devices required for a fibre-wireless base station. InP optoelectronic integrated circuit (OEIC) modules [6] avoid concerns with interconnections such as wire bonding or butt-coupling, and have the potential to yield reliable and cost-effective modules. OEICs may use a CPW transmission structure, so only single-sided wafer processing is required and the need for vias is eliminated.

In this Letter, a broadband millimetre-wave antenna fabricated on an InP wafer is presented, which is suitable for integration with microwave/optical circuits. The geometry investigated is the CPW-fed aperture coupled patch (ACP), which utilises the antenna design principles of [4] and [5].

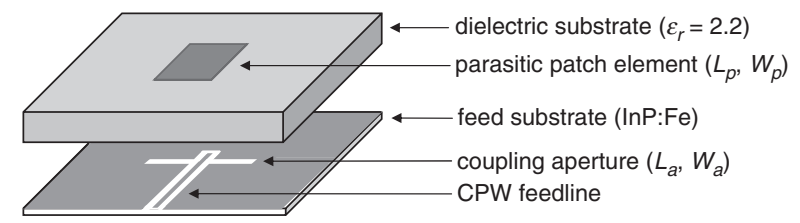

Fig. 1 Schematic of InP CPW-fed ACP

Dimensions: $L_{p}=2.5 \mathrm{~mm}, W_{p}=1 \mathrm{~mm}, L_{a}=2 \mathrm{~mm}, W_{a}=0.06 \mathrm{~mm}$

Antenna configuration: The general configuration of the InP CPWfed ACP is shown in Fig. 1. The feed substrate is a $350 \mu \mathrm{m}$-thick semi-insulating InP:Fe wafer, with a resistivity in excess of $10^{7} \Omega \mathrm{cm}$, and a relative permittivity of approximately 12.4 . A Ti-Ni-Au seed layer is deposited on the wafer to ensure the adhesion of the conducting layer to the InP. The CPW feedline and coupling aperture are then patterned and electroplated to a thickness of $8 \mu \mathrm{m}$. The InP:Fe wafer is mounted on an acrylic carrier, residing over a $1 \mathrm{~mm}$ deep well of RF absorber to nullify some of the rear-directed radiation. Rearward radiation can be significant when employing resonant aperture coupling to enhance the bandwidth (as seen in [4]). A photograph of the processed InP wafer mounted on the acrylic carrier is shown in Fig. 2.

The resonant aperture is mutually coupled to the parasitic patch element, which is fabricated on a $0.762 \mathrm{~mm}$-thick Rogers RT/Duroid 5880 substrate and placed centrally above the aperture. The parasitic patch and aperture dimensions are given in the caption to Fig. 1. The combination of the high-permittivity InP:Fe wafer and the low- permittivity RT/Duroid 5880 substrate form the hi-lo architecture, which has been proven to deliver efficient broadband antennas [5]. However, previous hi-lo antenna configurations have not incorporated semiconductor materials.

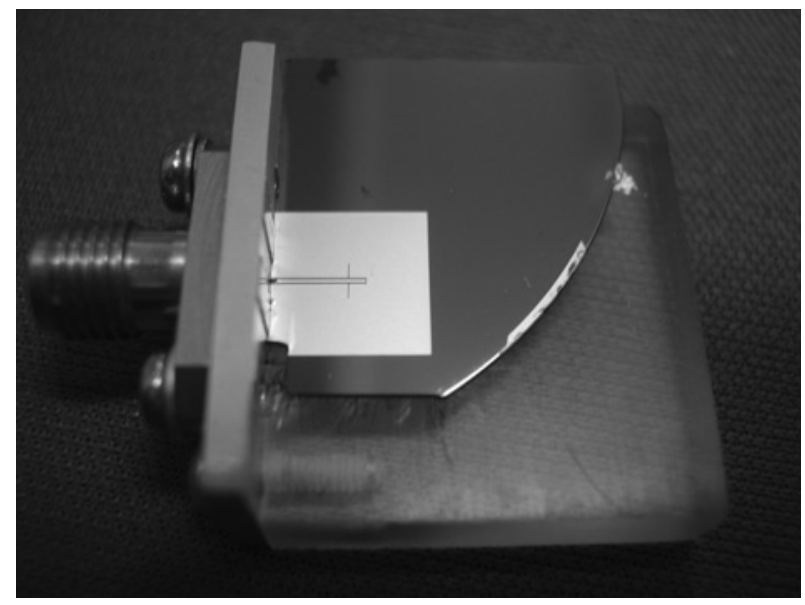

Fig. 2 Photograph of InP CPW-fed substrate mounted on acrylic carrier

Results and discussion: The measured return loss of the InP CPW-fed ACP can be derived from Fig. 3. The antenna exhibits an impedance bandwidth of $11.8 \%$ for $\operatorname{VSWR}<2$. We believe this to be the widest reported bandwidth for an antenna on InP. Although other ACP designs have achieved bandwidths in excess of $20 \%$, this has been achieved through the use of only low-permittivity materials. If desired, further bandwidth enhancement can be achieved by stacking an additional parasitic radiator over the InP CPW-fed ACP structure, as was done in [4].

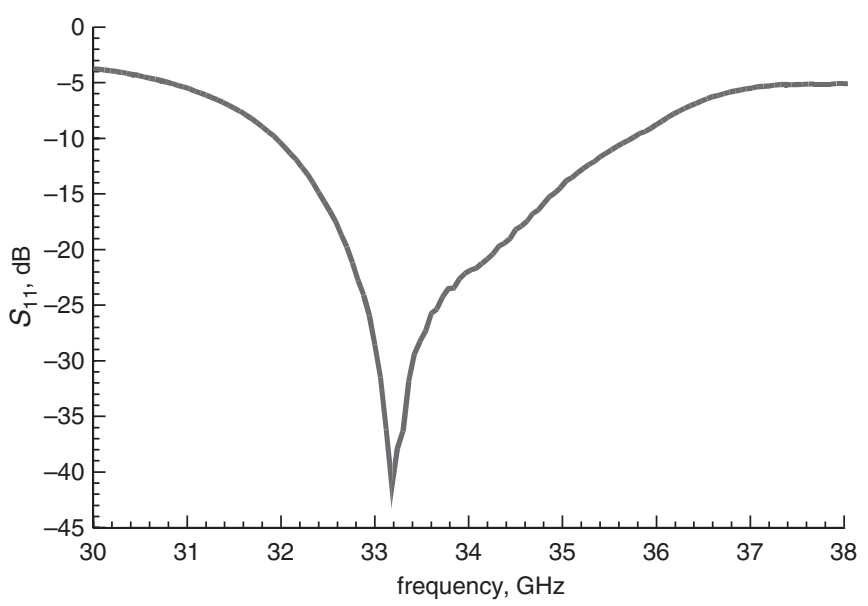

Fig. 3 Measured $S_{11}$ of InP CPW-fed $A C P$

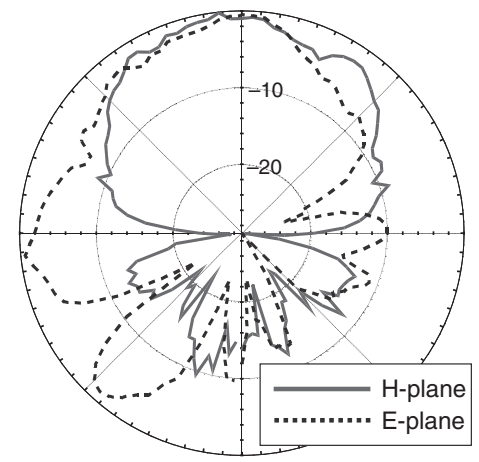

Fig. 4 Measured radiation patterns of InP CPW-fed ACP at $34 \mathrm{GHz}$

The far-field radiation patterns were measured at $34 \mathrm{GHz}$ and are shown in Fig. 4. The rough appearance of the radiation pattern is due to the manual measurement method in which readings were taken every 
$3^{\circ}$. A large sidelobe level is evident in the E-plane pattern. This is a consequence of field reflections from the brass plate used to attach the coaxial connector to the side of the acrylic carrier shown in Fig. 2. The maximum gain was measured to be approximately $8 \mathrm{dBi}$ close to the centre of the impedance bandwidth. The gain diminishes slowly towards the edges of the antenna bandwidth, and falls sharply for frequencies out-of-band. It is postulated that this is due to significant surface-wave generation outside the impedance bandwidth of the antenna as the energy is no longer strongly coupled to the parasitic patch element. Another effect of this surface-wave generation is the high out-of-band return loss of 4-5 dB.

Conclusions: An InP-based aperture-coupled printed antenna fed by a CPW has been presented. The antenna exhibits an impedance bandwidth of approximately $12 \%(\mathrm{VSWR}<2)$ and a measured gain of $8 \mathrm{dBi}$. The wide bandwidth and high gain make this antenna solution applicable to millimetre-wave fibre wireless transmitter/receiver modules, and other communications scenarios requiring efficient active integrated antennas.

(C) The Institution of Engineering and Technology 2006 19 April 2006

Electronics Letters online no: 20061253

doi: $10.1049 / \mathrm{el}: 20061253$
W.S.T. Rowe and K. Ghorbani (School of Electrical and Computer Engineering, RMIT University, GPO Box 2476V, Melbourne, Victoria, 3001, Australia)

E-mail:wayne.rowe@rmit.edu.au

\section{References}

1 Ahmed, Z., Novak, D., Waterhouse, R.B., and Liu, H-F.: '37-GHz fiberwireless system for distribution of broad-band signals', IEEE Trans. Microw. Theory Tech., 1997, MTT-45, (8), pp. 1431-1435

2 Qian, Y., and Itoh, T.: 'Progress in active integrated antennas and their applications', IEEE Trans. Microw. Theory Tech., 1998, MTT-46, (11), pp. $1891-1900$

3 Mirshekar-Syahkal, D., and Wake, D.: 'Bow-tie antennas on high dielectric substrates for MMIC and OEIC applications at millimetrewave frequencies', Electron. Lett., 1995, 31, (24), pp. 2060-2061

4 Rowe, W.S.T., and Waterhouse, R.B.: 'Broadband CPW fed stacked patch antenna', Electron. Lett., 1999, 35, (9), pp. 681-682

5 Rowe, W.S.T., Waterhouse, R.B., Nirmalathas, A., and Novak, D.: 'Integrated antenna base station design for hybrid fibre radio networks'. Int. Top. Meet. on Microwave Photonics, Melbourne, Australia, November 1999, pp. 47-50

6 Engel, Th., Strittmatter, A., Passenberg, W., Umbach, A., Schlaak, W., Dröge, E., Seeger, A., Steingrüber, R., Mekonnen, G.G., Unterbörsch, G. Bach, H.-G., Böttcher, E.H., and Bimberg, D.: 'Narrow-band photoreceiver OEIC on InP operating at $38 \mathrm{GHz}$ ', IEEE Photonics Technol. Lett., 1998, 10, (9), pp. 1298-1300 